\title{
CARBAMATO DE ETILA EM BEBIDAS ALCOÓLICAS (CACHAÇA, TIQUIRA, UÍSQUE E GRAPA)
}

\author{
Luiz Gualberto de Andrade-Sobrinho, Maurício Boscolo, Benedito dos Santos Lima-Neto e Douglas Wagner Franco* \\ Instituto de Química de São Carlos, Universidade de São Paulo, Av. do Trabalhador Sancarlense, 400, 13560-970 São Carlos - SP
}

Recebido em 19/9/01; aceito em 6/5/02

\begin{abstract}
ETHYL CARBAMATE IN ALCOHOLIC BEVERAGES (CACHAÇA, TIQUIRA, WHISKY AND GRAPE). The presence of ethyl carbamate in cachaças, tiquiras, whiskies and grapes was investigated by GC-MS, monitoring the m/z 62 ion. The external standard method was used for quantitation in 188 samples (126 cachaças, 37 tiquiras, 6 grappas and 19 whiskies). The results of the study for cachaça were analysed considering the geographic origin, distillation type (still or column), bottle coloration (amber or transparent) and ageing. The average contents of ethyl carbamate in cachaças and tiquiras were 0.77 and $2.4 \mathrm{mg} \mathrm{L}^{-1}$, respectively. These values were higher than the mean concentration found in grapes $\left(0.045 \mathrm{mg} \mathrm{L}^{-1}\right)$ and whiskies $\left(0.14 \mathrm{mg} \mathrm{L}^{-1}\right)$. On average the cachaças distilled in column tend to present higher values of ethyl carbamate than those from still. In the sampling studied it was not possible to define a correlation between content of ethyl carbamate, bottle coloration and ageing time.
\end{abstract}

Keywords: GC-MS; ethyl carbamate; alcoholic beverages; spirits.

\section{INTRODUÇÃO}

Carbamato de etila $\left(\mathrm{EtOCONH}_{2}\right)$ ou uretana é um composto potencialmente carcinogênico ${ }^{1}$ e forma-se naturalmente em alimentos tais como pão, iogurte, vinho, cerveja, saquêt $\hat{e}^{2-4} \mathrm{e}$, principalmente, em bebidas fermento-destiladas ${ }^{2-5}$ tais como uísque, rum, vodca, grapa, cachaça ${ }^{6,7}$ e tiquira (aguardente de mandioca) ${ }^{8}$.

Sendo o Brasil um dos maiores produtores de destilados alcoólicos do mundo9 ${ }^{9}$ é muito importante o conhecimento dos níveis de ocorrência de uma substância potencialmente carcinogênica como o carbamato de etila em tais produtos, pois além dos aspectos ligados à saúde pública, a sua presença em concentrações superiores a 0,150 $\mathrm{mg} \mathrm{L}^{-1}$ constitui também uma barreira para exportações para a Europa e América do Norte 5 .

Existem várias vias possíveis para a formação de carbamato de etila nas bebidas destiladas, geralmente envolvendo a reação entre o etanol e precursores nitrogenados, tais como uréia ${ }^{2}$, fosfato de carbamila $^{5}$ e cianeto $^{10}$. Este último é considerado um precursor de carbamato de etila durante e após o processo de destilação ${ }^{10}$.

O Canadá, em 1985, através do "Health and Welfare Department"s, estabeleceu os seguintes teores máximos de carbamato de etila para bebidas alcoólicas: vinhos $0,030 \mathrm{mg} \mathrm{L}^{-1}$; vinhos fortificados $0,100 \mathrm{mg}$ $\mathrm{L}^{-1}$; bebidas destiladas $0,150 \mathrm{mg} \mathrm{L}^{-1}$; destilados de frutas e licores 0,400 $\mathrm{mg} \mathrm{L}^{-1}$. A comparação entre os teores de carbamato de etila em bebidas alcoólicas brasileiras com os limites estabelecidos pelo Canadá é inevitável, em função de este ser, até o momento, o único país a possuir legislação específica sobre o assunto, sendo um referencial para os EUA e para a Comunidade Européia. Em março de 1990, o FDA ("U. S. Food and Drug Administration") publicou nota ${ }^{11}$ na qual os produtores de uísques americanos se comprometeram, a partir daquela data, a limitar o teor de carbamato de etila em $0,125 \mathrm{mg} \mathrm{L}^{-1}$.

Muitas metodologias analíticas vêm sendo propostas para a determinação de carbamato de etila em alimentos, todas empregando a cromatografia a gás com diferentes dispositivos de detecção ${ }^{12-19}$.

Em função das baixas concentrações em que se encontra o carbamato de etila nas bebidas alcoólicas e da baixa sensibilidade que alguns detectores apresentam, faz-se necessário além da con-

*e-mail: douglas@iqsc.sc.usp.br centração das amostras uma etapa de desidratação dos extratos, geralmente com $\mathrm{Na}_{2} \mathrm{SO}_{4}$ anidro ${ }^{14,15,20}$, tornando estes procedimentos morosos e mais susceptíveis a erros.

A cromatografia a gás acoplada à espectrometria de massas (GCMS) torna-se muito conveniente para a determinação de carbamato de etila em bebidas destiladas, pois além da elevada sensibilidade ${ }^{5,18}$ quando se opera no modo SIM, não há a necessidade de se desidratar as amostras 5 .

No presente trabalho aplicou-se a cromatografia a gás acoplada à espectrometria de massas, na determinação de carbamato de etila em bebidas fermento-destiladas. Também efetuou-se a comparação dos teores deste contaminante entre os destilados nacionais e os destilados estrangeiros. Os resultados foram analisados considerandose fatores ligados à região, tipo de destilação, coloração da embalagem e tempo de envelhecimento.

\section{PARTE EXPERIMENTAL}

\section{Reagentes}

Todos os reagentes utilizados foram de grau analítico. O carbamato de etila foi adquirido da Sigma-Aldrich. Utilizou-se etanol Mallinckrodt e água destilada desionizada em sistema Milli-Q.

\section{Preparação das soluções padrão}

A partir de uma solução estoque de carbamato de etila $22,0 \mathrm{mg}$ $\mathrm{L}^{-1}$ em etanol/água (40:60 v/v) $)^{15}$, foram preparadas as soluções-padrão para a construção da curva analítica $\left(0,050-12,0 \mathrm{mg} \mathrm{L}^{-1}\right)$.

\section{Metodologia}

Foi utilizado um cromatógrafo Shimadzu GC 17-A, equipado com detector de massas Shimadzu QP-5050A operando no modo de impacto eletrônico com $70 \mathrm{eV}$, com o monitoramento de íon seletivo m/z 62 por ser o mais empregado nas determinações de carbamato de etila ${ }^{21}$. A coluna cromatográfica capilar de fase polar (polietilenoglicol esterificada) utilizada foi uma HP-FFAP (50 m x 0,20 mm x 0,33 $\mu \mathrm{m}$ espessura do filme da fase estacionária). Empregou-se a seguinte pro- 
gramação de temperatura para o forno: $90^{\circ} \mathrm{C}(2 \mathrm{~min})$, elevada a $10^{\circ} \mathrm{C} /$ min até $150^{\circ} \mathrm{C}(0 \mathrm{~min})$, e então elevada a $40^{\circ} \mathrm{C} / \mathrm{min}$ até $230^{\circ} \mathrm{C}(10$ $\mathrm{min})$. As temperaturas do injetor e da interface do detector foram de 250 e $230^{\circ} \mathrm{C}$, respectivamente. $\mathrm{O}$ volume de injeção foi de $2,0 \mu \mathrm{L}$ no modo "splitless". Gás de arraste: He com fluxo de 1,5 mL/min.

Testes preliminares revelaram que o carbamato de propila, geralmente usado como padrão interno na determinação de carbamato de etila ${ }^{6,22}$, em solução etanólica pode sofrer trans-esterificação. Em vista disto, optou-se pelo emprego do método do padrão externo para quantificação. Os coeficientes de correlação das curvas analíticas $\left(1^{\circ}\right.$ grau $)$ variaram entre 0,9996 e $0,9998(\mathrm{~N}=6)$.

O limite de quantificação e a repetibilidade da metodologia utilizada foram estimados de acordo com a literatura ${ }^{23}$, sendo que o limite de quantificação foi de $10 \mu \mathrm{g} \mathrm{L}^{-1}$ e repetibilidade $(\mathrm{n}=10) 94,6 \%$.

\section{Amostras analisadas}

Foram analisadas em duplicata 126 amostras de aguardente de cana, sendo 63 da região sudeste, 39 da nordeste, 22 da sul e 2 da centro-oeste, bem como 37 amostras de tiquira, 6 amostras de grapas ( 1 brasileira e 5 italianas) e 19 amostras de uísque importados (6 americanos e 13 escoceses).

Procurou-se diversificar as origens das cachaças segundo a importância de cada Estado produtor (em volume e tradição de produção) e a disponibilidade de tais produtos. Os resultados encontrados para os Estados de Alagoas, Bahia, Maranhão, Espírito Santo, Goiás e Rio Grande do Sul podem não refletir o quadro real, em virtude da baixa representatividade do número de amostras analisadas.

As tiquiras eram provenientes do Estado do Maranhão e as grapas, de origem italiana, foram fornecidas cordialmente pela "Associazione Nazionale Assaggiatori Grappa e Acqueviti-ANAG” (Brescia - Itália), enquanto a grapa brasileira, de característica artesanal, foi adquirida no Estado de Santa Catarina.

Foram também analisadas 21 amostras de cachaças envelhecidas de reservas pessoais gentilmente cedidas por produtores de aguardente de cana. Em alguns casos, mais de uma amostra de uma mesma cachaça comercial foi analisada.

\section{RESULTADOS E DISCUSSÃO}

A Figura 1(a) mostra o espectro de massas por impacto eletrônico do carbamato de etila e nas Figuras 1(b) e 1(c) são apresentados cromatogramas referentes a uma solução-padrão $\left(0,8 \mathrm{mg} \mathrm{L}^{-1}\right) \mathrm{de}$ carbamato de etila e de uma amostra de aguardente de cana, respectivamente. Observa-se que, pelo monitoramento do íon $\mathrm{m} / \mathrm{z} 62$, é possível identificar e quantificar o carbamato de etila sem problemas de co-eluições com outros compostos presentes na cachaça.

Nas 126 amostras de cachaça analisadas, o teor médio de carbamato de etila foi $0,77 \mathrm{mg} \mathrm{L}^{-1}$, variando de 0,013 a $5,7 \mathrm{mg} \mathrm{L}^{-1}$. A distribuição das cachaças em função do teor de carbamato de etila é mostrada na Figura 2. Apenas $21 \%$ das amostras analisadas apresentaram teores $\leq 0,150 \mathrm{mg} \mathrm{L}^{-1}$, valor considerado internacionalmente aceitável ${ }^{5}$.

Na Figura 3 apresenta-se a distribuição das aguardentes segundo o teor médio de carbamato de etila por Estado produtor. Dentre os Estados mais representativos, em termos de produção de cachaça, o menor teor médio de carbamato de etila foi encontrado nas amostras provenientes do Ceará $\left(0,44 \mathrm{mg} \mathrm{L}^{-1}\right)$, enquanto que Minas Gerais foi o que apresentou o maior $\left(1,00 \mathrm{mg} \mathrm{L}^{-1}\right)$, seguido por São Paulo $\left(0,87 \mathrm{mg} \mathrm{L}^{-1}\right)$ e Pernambuco $\left(0,83 \mathrm{mg} \mathrm{L}^{-1}\right)$. Esta informação sugere a necessidade de se iniciar um processo de rastreamento das diferentes práticas regionais de produção de cachaça, para melhor conhecer os fatores responsáveis pela formação de carbamato de etila.
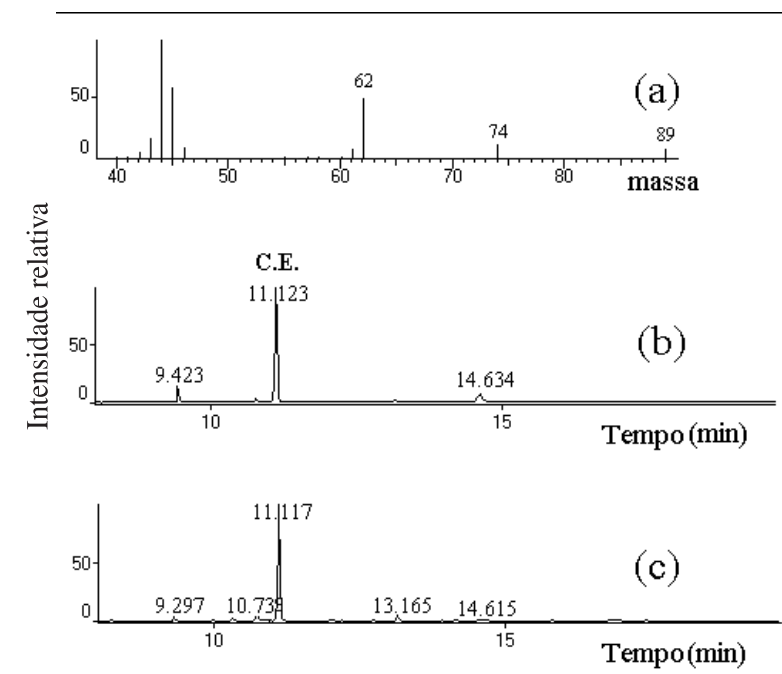

Figura 1. (a) Espectro de massas do carbamato de etila. Cromatogramas por GC-MS (SIM, m/z = 62); (b) Solução-padrão de carbamato de etila (C.E.); (c) Amostra de cachaça

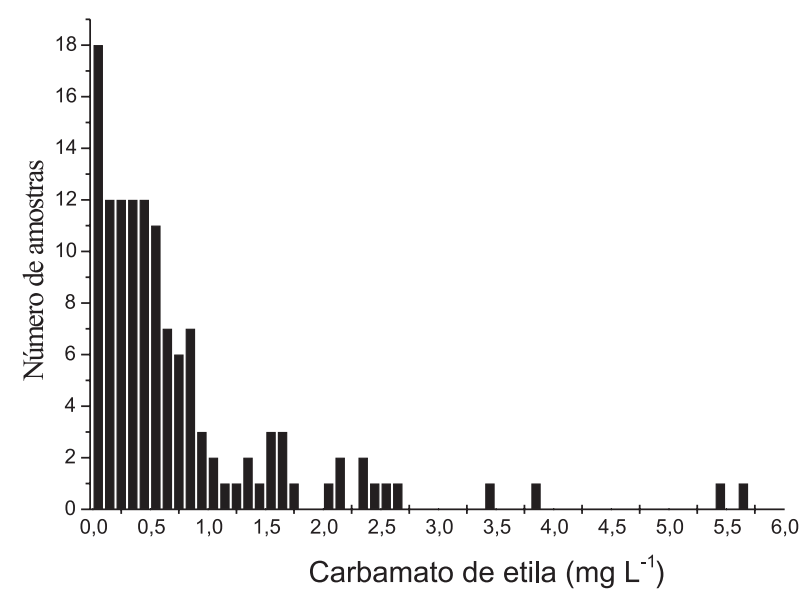

Figura 2. Distribuição das 126 amostras de cachaça em função do teor de carbamato de etila (intervalos de $0,10 \mathrm{mg} \mathrm{L}^{-1}$ )

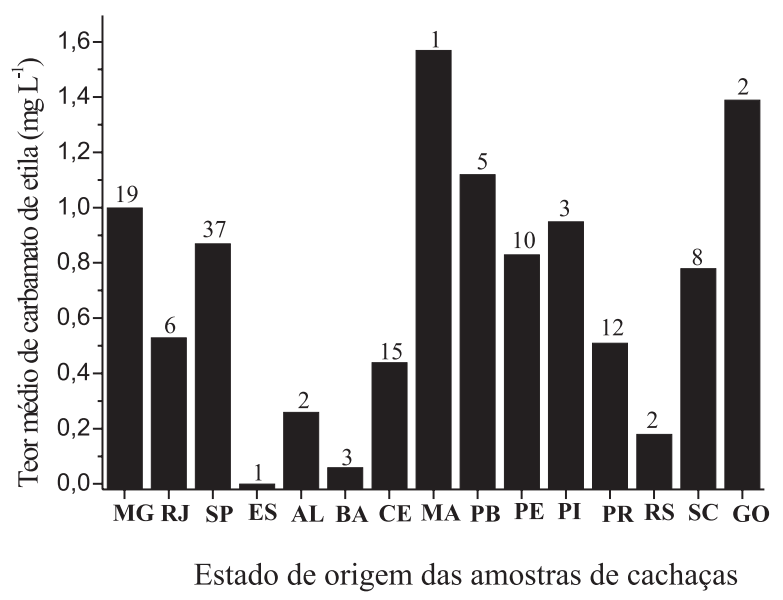

Figura 3. Teores médios de carbamato de etila em função da origem geográfica das cachaças analisadas. Os índices sobre as colunas indicam o número de amostras por Estado 
Como o teor de carbamato de etila depende da disponibilidade de seus precursores na matéria-prima processada, e sendo todas as cachaças derivadas da mesma matéria-prima, um dos objetivos deste trabalho foi conhecer os vetores do processo de produção desta bebida que influenciam na formação deste contaminante.

Um dos fatores investigados foi a influência do tipo de destilação da cachaça na formação de carbamato de etila. Sabe-se que com a necessidade de volumes de produção cada vez maiores, muitos produtores abandonaram o sistema de destilação tradicional em alambique (sistema descontínuo) e adotaram a destilação em coluna (sistema contínuo). Contatando os produtores de cachaça, foi possível identificar que, dentre as amostras estudadas, 34 foram destiladas em alambique e 69 em coluna. Para as demais 23 amostras não foi possível obter esta informação.

Pode-se observar na Figura 4 que as amostras destiladas em alambique apresentaram um maior percentual de amostras com teor de carbamato de etila $\leq 0,150 \mathrm{mg} \mathrm{L}^{-1}$. Nas faixas de concentração 0,401 $1,00 \mathrm{mg} \mathrm{L}^{-1}$ e acima de $1,00 \mathrm{mg} \mathrm{L}^{-1}$ notou-se um crescente aumento percentual das amostras destiladas em coluna. Os teores médios de carbamato de etila nas amostras destiladas em alambique e coluna foram de 0,63 e $0,93 \mathrm{mg} \mathrm{L}^{-1}$, respectivamente.

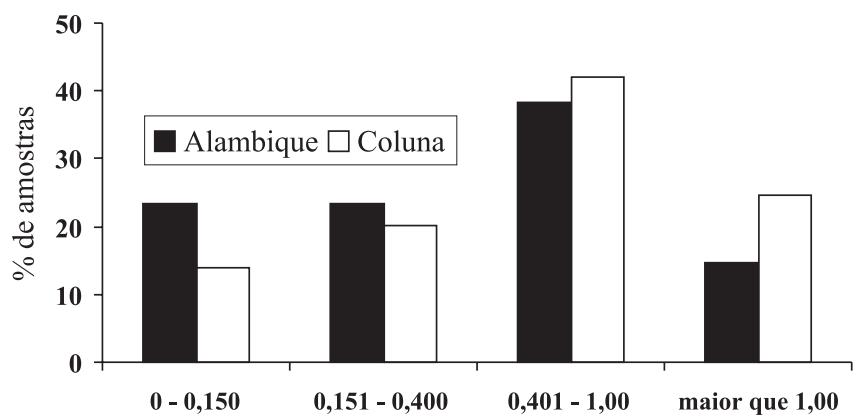

Faixa de concentração de carbamato de etila $\left(\mathrm{mg} \mathrm{L}^{-1}\right)$

Figura 4. Distribuição das amostras de cachaças em função da faixa de concentração de carbamato de etila e do sistema de destilação

Isto provavelmente é consequiência não apenas do sistema de destilação em si, mas também da natureza do material utilizado na confecção dos aparelhos destiladores. Os alambiques, em sua grande maioria, são construídos em cobre e as colunas, em aço inoxidável.

A escolha do aço inoxidável para a construção das colunas de destilação deve-se ao fato que este material, não sofrendo corrosão, não causa problemas de manutenção. Entretanto, as bebidas destiladas em aparelhos construídos exclusivamente em aço inoxidável, apresentam características sensoriais desagradáveis ${ }^{24}$, principalmente devido aos altos teores de dimetil sulfeto liberado no processo e que permanece no produto final ${ }^{25}$.

Com o emprego de partes em cobre no aparelho destilador, as propriedades organolépticas negativas são notadamente diminuídas, em função da influência deste metal na conversão do dimetil sulfeto a sulfato ${ }^{25}$. Desta forma, um expediente normalmente empregado pelos produtores de cachaça que operam em modo contínuo é a adoção de condensadores de cobre (parte descendente do fluxo) no aparelho destilador.

A associação destes materiais (aço inoxidável e cobre) é eficiente na solução dos problemas sensoriais dos destilados mas, por outro lado, pode favorecer consideravelmente a formação de carbamato de etila, uma vez que o ácido cianídrico, não sendo fixado na coluna (parte ascendente do fluxo) atinge o condensador, complexando-se com os íons $\mathrm{Cu}$ (II) originados da corrosão do condensador pelos vapores ácidos da bebida. Através da redução do $\mathrm{Cu}(\mathrm{II})$ a $\mathrm{Cu}(\mathrm{I})$ e com a formação de cianogênio, seguido de seu desproporcionamento, ocorre a formação de íons cianato ${ }^{26}$.

$$
\begin{aligned}
& 2 \mathrm{Cu}(\mathrm{II})+4 \mathrm{CN}^{-} \rightarrow 2 \mathrm{Cu}(\mathrm{CN})_{2} \\
& 2 \mathrm{Cu}(\mathrm{CN})_{2} \rightarrow 2 \mathrm{CuCN}+\mathrm{C}_{2} \mathrm{~N}_{2} \\
& \mathrm{C}_{2} \mathrm{~N}_{2}+2 \mathrm{OH}^{-} \rightarrow \mathrm{NCO}^{-}+\mathrm{CN}^{-}+\mathrm{H}_{2} \mathrm{O}
\end{aligned}
$$

Os íons cianato por reação com etanol produzem o carbamato de etila $^{27}$.

$\mathrm{NCO}^{-}+\mathrm{EtOH} \rightarrow \mathrm{EtOCONH}_{2}$

Quando cobre é empregado na parte ascendente do fluxo, como ocorre nos alambiques, é esperado que ocorra uma fixação de cianeto $^{28}$, com a formação compostos tais como: $\mathrm{CuCN}, \mathrm{Cu}(\mathrm{CN})_{2}$, $\mathrm{Cu}_{2}(\mathrm{CN})_{3}^{-}, \mathrm{Cu}_{3}(\mathrm{CN})_{4}^{-}$, diminuindo a concentração de cianeto no destilado $^{7}$ e consequientemente, reduzindo o teor de carbamato de etila.

Outro fator a ser considerado é a radiação luminosa ${ }^{29,30}$, que pode exercer uma influência na formação de carbamato de etila após a destilação, favorecendo a oxidação radicalar do cianeto a cianato e, assim, formando carbamato de etila (Eq. 4). Considerando-se que a parede dos recipientes pode agir como um filtro à radiação luminosa, procurou-se uma possível correlação entre a coloração das garrafas e o teor de carbamato de etila dos destilados. O teor médio determinado para as amostras engarrafadas em recipientes âmbar (21 amostras) foi de $0,83 \mathrm{mg} \mathrm{L}^{-1}$, variando de 0,09 a $3,5 \mathrm{mg} \mathrm{L}^{-1}$ enquanto que em garrafas transparentes (105 amostras) foi de $0,72 \mathrm{mg} \mathrm{L}^{-1}$ variando de 0,01 a 5,7 $\mathrm{mg} \mathrm{L}^{-1}$.

Não se observou uma diferença significativa entre a coloração das garrafas e o teor de carbamato de etila. Tal resultado indica que a formação de carbamato de etila após o engarrafamento, se ocorrer em proporções consideráveis, é independente da radiação luminosa incidente sobre a bebida. Vários fatores são importantes para a formação de carbamato de etila em bebidas destiladas, porém ainda não existe uma explicação satisfatória sobre a sua influência nas cachaças. Em estudo anterior ${ }^{31}$, investigou-se a correlação entre o teor de carbamato de etila em cachaças e a presença de alguns de seus precursores, como uréia, cianeto e aminoácidos. Apenas para o íon cianeto foi possível observar uma tendência à correlação positiva.

Existe ainda um terceiro fator que é o tempo de envelhecimento da cachaça, porém não se verificou correlação evidente entre o teor de carbamato de etila e o tempo de envelhecimento das amostras. Nas amostras envelhecidas analisadas, o teor médio de carbamato de etila foi de $0,69 \mathrm{mg} \mathrm{L}^{-1}$, variando de não detectado (N.D.) até $2,6 \mathrm{mg} \mathrm{L}^{-1}$, enquanto que para as amostras não envelhecidas a concentração média foi de $0,87 \mathrm{mg} \mathrm{L}^{-1}$, variando de 0,04 a $5,7 \mathrm{mg} \mathrm{L}^{-1}$. Estas informações sugerem que a formação de carbamato de etila a partir de seus precursores deve ocorrer principalmente após um curto intervalo de tempo depois da destilação.

As amostras de tiquira analisadas apresentaram teor médio de carbamato de etila (2,4 $\left.\mathrm{mg} \mathrm{L}^{-1}\right)$ muito superior ao da cachaça (Tabela 1). Observou-se que as amostras de tiquira com a concentração de carbamato de etila inferior a $0,65 \mathrm{mg} \mathrm{L}^{-1}$ apresentaram graduação alcoólica $\leq 28 \%$ Vol., graduação esta bem abaixo do mínimo especificado pelo Ministério da Agricultura e do Abastecimento ${ }^{32}$ que é de $38 \%$ Vol.

Atualmente, estão em curso investigações para se determinar os fatores responsáveis pelo alto teor de carbamato de etila nas cachaças e nas tiquiras. Estes estudos estão focalizados tanto no processo fermentativo como durante e após o processo de destilação (cachaça e tiquira).

Nas grapas e uísques (Tabela 1) observou-se um teor médio de carbamato de etila inferior ao dos destilados tipicamente brasileiros. 
Tabela 1. Concentração de carbamato de etila em tiquiras, grapas e uísques

\begin{tabular}{|c|c|c|c|c|c|}
\hline Amostra & Carbamato de etila $\left(\mathrm{mg} \mathrm{L}^{-1}\right)$ & Amostra & Carbamato de etila $\left(\mathrm{mg} \mathrm{L}^{-1}\right)$ & Amostra & Carbamato de etila $\left(\mathrm{mg} \mathrm{L}^{-1}\right)$ \\
\hline T 01 & 10 & T 22 & 2,7 & G 06 & 0,068 \\
\hline Т 02 & 1,3 & Т 23 & 2,3 & UA 01 & 0,075 \\
\hline T 03 & 1,0 & T 24 & 2,1 & UA 02 & 0,40 \\
\hline Т 04 & 5,2 & Т 25 & $0,21(0,40)^{*}$ & UA 03 & 0,089 \\
\hline Т 05 & 3,9 & T 26 & $0,20(0,37)^{*}$ & UA 04 & 0,19 \\
\hline Т 06 & 0,83 & Т 27 & $0,20(0,37)^{*}$ & UA 05 & 0,064 \\
\hline Т 07 & 2,7 & T 28 & $0,19(0,36)^{*}$ & UA 06 & 0,15 \\
\hline T 08 & 3,1 & Т 29 & 1,8 & UE 01 & 0,10 \\
\hline Т 09 & 3,4 & T 30 & $0,20(0,48) *$ & UE 02 & 0,12 \\
\hline T 10 & 6,1 & T 31 & $0,19(0,46)^{*}$ & UE 03 & 0,34 \\
\hline T 11 & 3,8 & T 32 & 3,1 & UE 04 & 0,089 \\
\hline T 12 & $0,65(1,1)^{*}$ & T 33 & 1,1 & UE 05 & 0,12 \\
\hline T 13 & 5,6 & Т 34 & 1,2 & UE 06 & 0,11 \\
\hline Т 14 & 1,3 & Т 35 & 1,5 & UE 07 & 0,089 \\
\hline T 15 & 2,9 & Т 36 & 2,4 & UE 08 & 0,19 \\
\hline T 16 & 1,7 & Т 37 & 1,4 & UE 09 & 0,19 \\
\hline T 17 & 1,9 & G 01 & N. D. & UE 10 & 0,068 \\
\hline T 18 & 2,5 & G 02 & N. D. & UE 11 & 0,094 \\
\hline Т 19 & 1,0 & G 03 & 0,025 & UE 12 & 0,073 \\
\hline Т 20 & 1,3 & G 04 & 0,041 & UE 13 & 0,065 \\
\hline Т 21 & 6,1 & G 05 & N. D. & & \\
\hline
\end{tabular}

(T) tiquira; (G) grapa; (UA) uísque americano; (UE) uísque escocês; (N. D.) Não detectado.

* Os valores entre parênteses correspondem às concentrações estimadas considerando-se uma correção para o grau alcoólico médio de $48 \%$ Vol.

As grapas, tanto as italianas como a brasileira, apresentaram teor médio de $0,045 \mathrm{mg} \mathrm{L}^{-1}$. No caso dos uísques, embora $25 \%$ das amostras analisadas apresentem teores acima de $0,150 \mathrm{mg} \mathrm{L}^{-1}$, o teor médio determinado foi de $0,14 \mathrm{mg} \mathrm{L}^{-1}$.

Cumpre ressaltar que no passado, também os uísques apresentavam elevados teores de carbamato de etila ${ }^{3}$. Modificações no processo de produção, estimuladas por legislação específica e pressão do mercado consumidor influenciaram na melhoria da qualidade do produto final ${ }^{33}$. Tal fato ocorreu na Itália com a grapa e também pode vir a ocorrer no Brasil.

Por ser o carbamato de etila uma substância de potencial carcinogênico, estes resultados indicam a necessidade urgente de alterações no processo de produção de aguardente de cana e tiquira, tanto para eliminar este problema de saúde pública, bem como para o enquadramento destes produtos nos padrões internacionais.

Um fato importante que deve ser realçado é que estes resultados referem-se às bebidas disponíveis no mercado. Somente o acompanhamento da bebida ao longo do processo de produção e armazenagem irá permitir uma melhor avaliação da gênese do carbamato de etila em cachaças e tiquiras.

\section{AGRADECIMENTOS}

Os autores agradecem ao Dr. L. Odello do Centro Studi Assagiatori de Brescia (Itália) pela doação das amostras de grapas e também à CAPES, CNPq e FAPESP (proc. 98/16038-5 ) pelo apoio financeiro.

\section{REFERÊNCIAS}

1. Schlatter, J.; Lutz, W. K.; Food Chem. Toxicol. 1990, 3, 205.

2. Zimmerli, B.; Schlatter, J.; Mutat. Res. 1991, 259, 325.

3. Battaglia, R.; Conacher, B. S.; Page, B. D.; Food Addit. Contam. 1990, 7 , 477.

4. Ough, C. S.; J. Agric. Food Chem. 1976, 24, 323.

5. Lawrence, J. F.; Page, B. D.; Conacher, H. B. S.; Adv. Environ. Sci. Technol. 1990, 23, 457.

6. Nagato, L. A. F.; Silva, O. A.; Yonamine, M.; Penteado, M. V. C.; Alimentaria 2000, 311, 31.

7. Boscolo, M.; Tese de Doutorado, Universidade de São Paulo, Brasil, 2001.
8. Boscolo, M.; Andrade-Sobrinho, L.G.; Lima-Neto, B. S.; Marques, E. P.; Franco, D. W.; Engarrafador Moderno 1998, 58, 62.

9. Lima-Neto, B. S.; Franco, D. W.; Engarrafador Moderno 1994, 33, 05.

10. Cook, R.; McCaig, N.; McMillan, J. M. B.; Lumsden, W. B.; J. Inst. Brew. 1990, 96, 233.

11. Dyer, R. H.; J. AOAC Int. 1994, 77, 64

12. Dennis, M. S.; Howart, N.; Massey, R. C. ; Parker, I.; Scotter, M.; Startin, J. R.; J. Chromatogr. 1986, 369, 193.

13. Walker, G.; Winterlin, W.; Fouda, H. N.; Seiber, J.; J. Agric. Food Chem. 1974, 22, 944.

14. Canas, B. J.; Havery, D. C.; Joe Jr., F. L.; J. Assn. Offic. Anal. Chem. 1988, $71,509$.

15. Ma, Ya-P.; Deng, Fu-Q.; Chen, D.-Z.; Sun, Shou-W.; J. Chromatogr., A 1995, 695, 259.

16. Joe, F. L.; Kline, D. A.; Miletta, E. M.; Roach, J. A.; Roseboro, E. L.; Fazio, T.; J. Assn. Offic. Anal. Chem. 1977, 60, 509.

17. Van Ingen, R. H.; Nijssen, L. M.; Berg, F.; Maarse, H.; J. High Res. Chromatogr. 1987, 10, 151.

18. Benjamin, P. Y.; Webwe, D.; Pages, B. D.; J. Chromatogr., A 1987, 402, 233.

19. Mossoba, M. M.; Chem, J. T.; Brumley, W. C.; Page, S. W.; Anal. Chem. 1988, 60, 945

20. Brumley, W. C.; Canas, B. J.; Perfetti, G. A.; Mossobe, M. M.; Sphon, J. A.; Cornelius, P. E.; Anal. Chem. 1988, 60, 975.

21. Clegg, B. S.; Frank, R.; J. Agric. Food Chem. 1988, 36, 502.

22. Canas, B. J.; Joe Jr., F. L.; Diachenko, G. W.; J. AOAC Int. 1994, 6, 1530.

23. McNair, H. M.: Miller, J. M.; Basic Gas Chromatography, John Wiley \& Sons: New York, 1998.

24. Faria, J. B.; Deliza, R.; Rossi, E. A.; Ciênc. Tecnol. Aliment. 1993, 13, 89.

25. Nascimento, R. F.; Tese de Doutorado, Universidade de São Paulo, Brasil, 1997.

26. Beattie, J.K.; Polyblank, G. A.; Aust. J. Chem. 1995, 48, 861.

27. Taki, N.; Imamura, L.; Takebe, S.; Kobashi, K.; Jpn. J. Toxicol. Environ. Health 1992, 8, 498.

28. MacKenzie, W. M.; Clyne, A. H.; MacDonald, L. S.; J. Inst. Brew. 1990, 96, 223.

29. Guerain, J.; Leblond, N. Em Elaboration et Connaissance des Spiriteux; Cantagrel, R., ed.; Tec\&Doc: Paris, 1992, p. 330.

30. Tegmo-Larsson, I. M.; Spittler, T. D.; J. Food Sci. 1990, 55, 1166.

31. Aresta, M.; Boscolo, M.; Franco, D. W.; J. Agric. Food Chem. 2001, 49, 2819.

32. MINISTÉRIO DA AGRICULTURA. Portaria $N^{\circ}$ 371. Complementação de Padrões de Identidade e Qualidade para Destilados Alcoólicos. Brasília, DF, (18/09/1974).

33. http://www.foodstandards.gov.uk/fsainfsheet/2000/n²/2 whisky.htm, acessada em Setembro 2001. 